\title{
$\phi$ \\ Placebo and nocebo effects in visual attention
}

\section{Introduction}

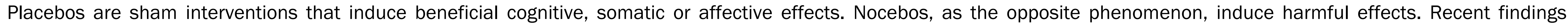

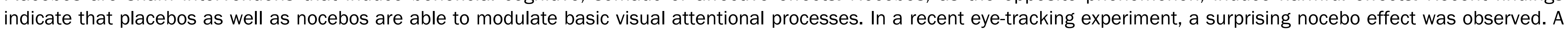

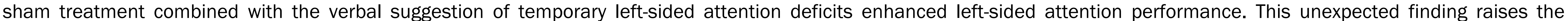

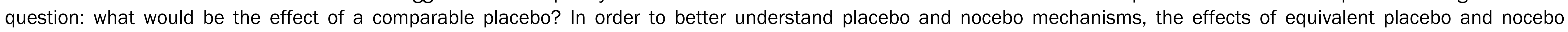
suggestions on visual-spatial attention were directly compared with each other in the present eye-tracking study.

Method
Design and Procedure
A visual search task was performed once without
(control condition) and once with the placebo or the
nocebo (placebo/nocebo condition). The search task had
three visual load conditions with either 50, 100, or
200 distractors. Participants were instructed to
find a single target as fast as possible (max. 90 s). In
each trial the target had a different position (Fig. 1).
Sample
Forty right-handed healthy women were analyzed
(age: $\mathrm{M}=21.06$ years, SD $=2.58$ ). The subjects either
participated in the placebo group ( $n=20$ ) or in the
nocebo group ( $n=20$ ). All participants reported to
experience symptoms in accordance with the
placebo/nocebo suggestion ('placebo/nocebo responder'; 1
(not at all) - 100\% (very effective); Nocebo group $=49.50 \%, \mathrm{SE}=3.18 ;$
Placebo group $=54.35 \%, \mathrm{SE}=4.03$; $\mathrm{p}>$.28).

\section{Placebo/Nocebo}

The placebo/nocebo was a sham transcranial magnetic stimulation (STMS), which was administered with the suggestion that the treatment would induce either temporary left-sided attention improvements (placebo) or deficits (nocebo). The sTMS system was administered for 4 min in the placebo/nocebo condition. After the stimulation, the system was removed and the visual search task started (Fig. 1).

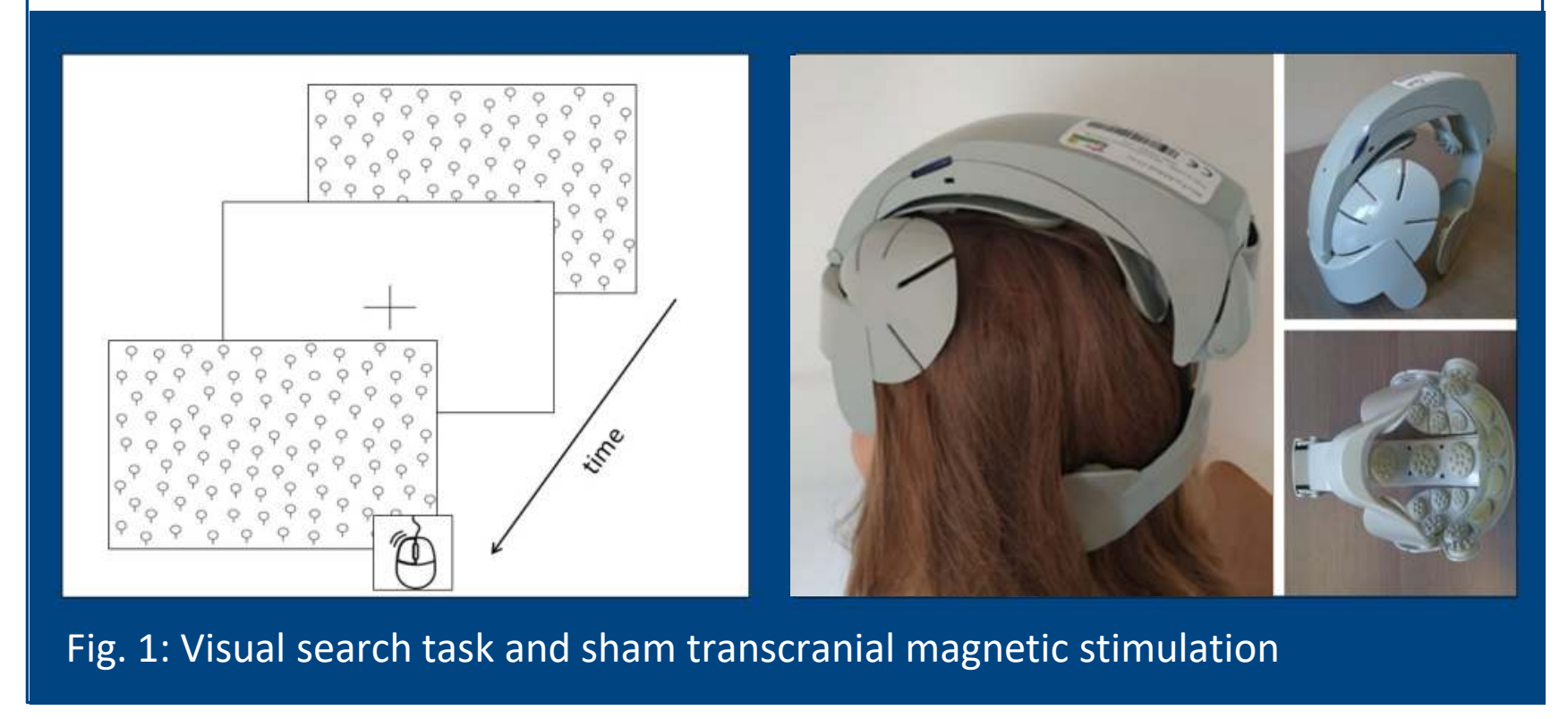

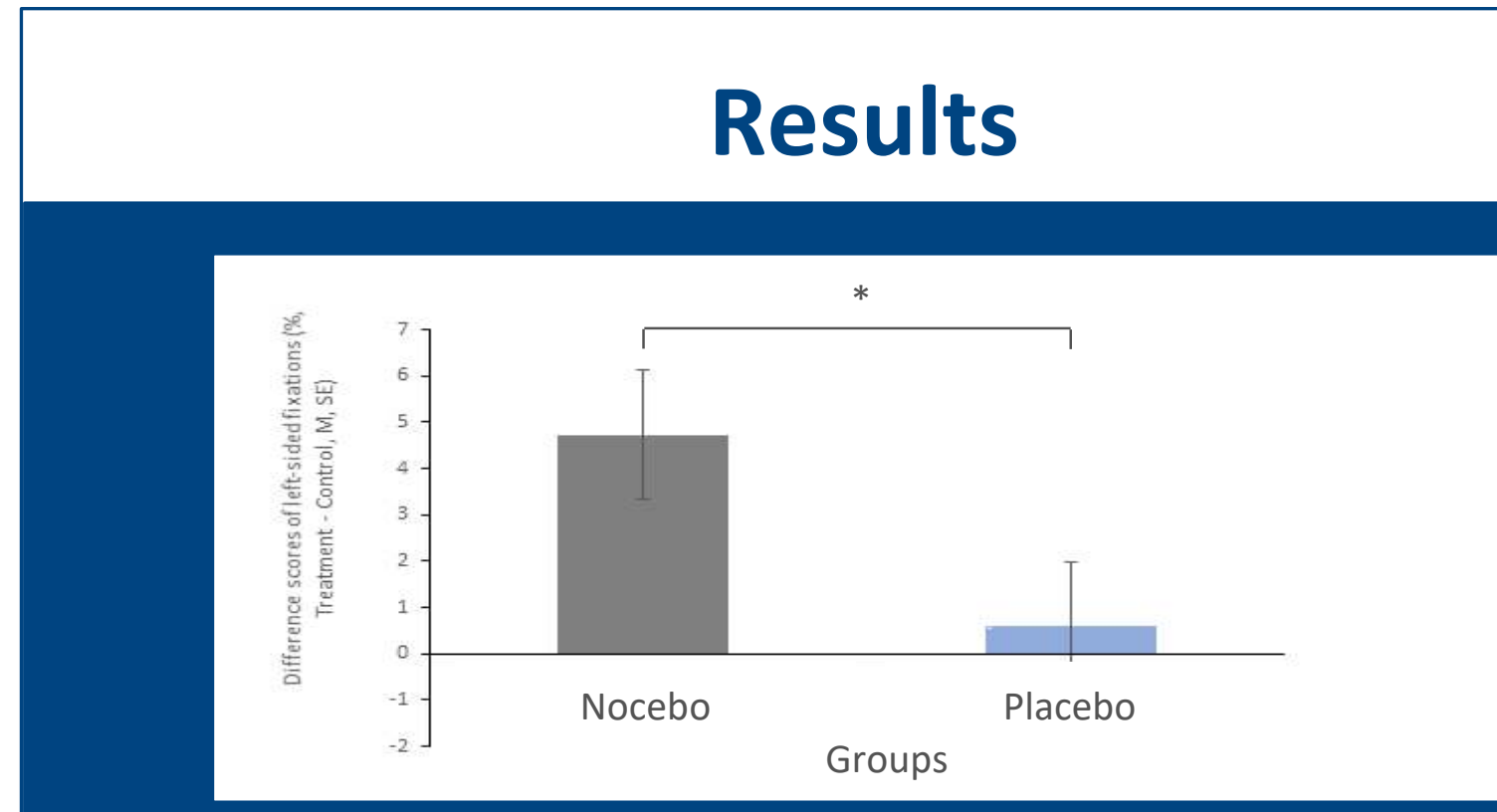

Fig. 2: Mean difference scores for percentages of left-sided fixations

Contrary to the verbal suggestion, the nocebo group showed more left-sided fixations and faster responses to left-sided targets in the nocebo condition compared to the control condition $(\mathrm{p}<$ .002). The placebo had no effect on unilateral fixations or reaction times $(p>.06)$. The nocebo group showed more left-sided fixations (independent of the visual load level, Fig. 2) and faster responses to leftsided targets in the high-load condition $(F(2,76)=$ $4.41, p=0.015$ ) than the placebo group.

\section{Discussion}

The present findings indicate a more beneficial effect of a nocebo compared to a placebo for the first time. Although, placebo responders subjectively experienced effects of the placebo, no objective effect was detected. This overestimation in the placebo group could have inhibited additional efforts. In contrast, the experience of attention deficits in the nocebo group could have promoted compensatory mechanisms and, finally, improved the actual performance. These results raise questions regarding the possibilities and limitations of placebos and nocebos. 